\title{
THE CLINICAL EFFICIENCY OF PLATELET RICH PLASMA IN THE TREATMENT OF TEMPOROMANDIBULAR JOINT DISORDERS
}

\author{
Elham F Hassan ${ }^{l} B D S$, Tarek M Ali ${ }^{2} P h D$, Nevein S Abdulla ${ }^{2} P h D$
}

\begin{abstract} PRP safety and efficacy in the management of musculo articular disorders. TMD. of 1 week, 3 , and 6 months. CONCLUSIONS: PRP injection is a safe and effective method in the treatment of TMDs.

KEY WORDS: TMJ, TMDs, PRP, Arthrocentesis.

1- Clinical Instructor in Oral \& Maxillofacial surgery department, Faculty of Dentistry, Asyut University

2- Professor of Oral \& Maxillofacial Surgery, Faculty of Dentist, Alexandria University.
\end{abstract}

INTRODUCTION: Temporomandibular joint dysfunction (TMD) is a clinically significant condition which can be a source of acute or chronic orofacial pain and dysfunction including limitation of mandibular movement. Platelet-rich plasma (PRP) is a blood product that allows in a simple, low cost, and minimally invasive way to obtain a concentration of many growth factors when activated. Many researchers proved

OBJECTIVES: to evaluate the efficiency of intra-articular PRP injection in comparison with conventional arthrocentesis in the treatment of

MATERIALS AND METHODS: Twenty patients suffering from TMD were included in the study diagnosed according to The Research Diagnostic Criteria for Temporomandibular disorders (RDC/TMD) and divided into two groups as follow; ten patients underwent intra-articular injections of $2 \mathrm{ml} \mathrm{PRP} \mathrm{into} \mathrm{the} \mathrm{temporomandibular} \mathrm{joint} \mathrm{(TMJ)} \mathrm{(study} \mathrm{group)} \mathrm{and} \mathrm{the} \mathrm{other} \mathrm{ten} \mathrm{patients} \mathrm{were} \mathrm{treated} \mathrm{by} \mathrm{the} \mathrm{conventional}$ arthrocentesis using Ringer's solution (control group). The patients were clinically evaluated preoperatively and postoperatively at the intervals

RESULTS: The differences between the two groups in all the measured parameters were statistically insignificant throughout the postoperative period. But PRP group showed superior result than arthrocentesis group regarding pain and tenderness, and inferior result in clicking.

\section{INTRODUCTION}

Temporomandibular joint (TMJ) is a compound joint, including the temporal bone, mandible, numerous associated muscles, a specialized fibrous tissue, articular disk, several ligaments and numerous associated muscles (1).

Temporomandibular joint disorders (TMDs) are a form of musculoskeletal pain of the TMJ and masticatory muscles of nonspecific etiology (2). A number of conservative methods is used in the treatment of TMDs including occlusal splints, supportive physical therapy procedures, rehabilitation involving muscular training, and psychological support $(3,4)$. Intra-articular administration of medications is an established method of treatment, particularly in orthopedic and rheumatic disorders associated with pain, effusion, inflammation of cartilage, bone and joint capsules as well as fibrous adhesions. Currently, agents used for intra- articular injection within the temporomandibular joint region include hyaluronic acid and steroids (5).

Platelet rich plasma (PRP) first was used in cardiac surgery by M. Ferrari in 1987(6); then it is being used as a treatment option for specialties including orthopedics, sports medicine, dentistry, ophthalmology, otolaryngology, neurosurgery, and maxillofacial surgery (7). PRP is a natural concentrate of blood growth factors and is thought to deliver activated platelets when injected thus may reduce inflammation, provide pain relief, improve function and stimulate possible cartilage regeneration at the site of injury $(8,9)$.

Filardo et al. (10) reported the use of platelet-rich plasma in the treatment of intra-articular cartilage injuries and knee inflammations; the treatment was reported to cause rapid reduction of pain and quick recovery of functional capability. Studies conducted by Sundman et al. (2) demonstrated significant anti-inflammatory properties of plasma.

Research findings derived from basic and preclinical studies and from clinical trials collectively suggested that PRP is a promising treatment for joints disorders and relieving symptoms owing to its biological properties as it has an anabolic effect on chondrocytes, Mesenchymal stem cell and synoviocytes with resultant increases in cell proliferation $(11,12)$, cartilaginous extracellular matrix accumulation, and increase hyaluronic acid secretion (12). PRP has the potential to inhibit inflammation and alleviate osteoarthritis symptoms with a clinically acceptable safety profile (13-15).

Many researchers referred the high efficacy of plateletrich plasma in the management of musculo-articular disorders and its safety to the use of autologous material as well as the low costs of treatment $(10,16,17)$.

This study was designed to evaluate the clinical efficacy of platelet rich plasma in the treatment of TMD in comparison with the conventional arthrocentesis.

\section{MATERIALS AND METHODS}

Twenty patients were selected from those attending the outpatient clinic of Oral and Maxillofacial Surgery department, Faculty of Dentistry, Alexandria University. Their age ranged from 25 to 50 years and diagnosis was made on the basis of Research Diagnostic Criteria for Temporomandibular Disorders (RDC/TMD).

The ethical clearance was obtained by the ethical committee before the study began and the selected patients were informed about the nature of the study and informed consents were signed.

Before starting any procedure, different conservative modalities were exhausted first including patient education 
and instructions for habit modification. Patients were instructed to use soft diet, moist heat application, analgesic anti-inflammatory drugs and splint therapy. When the patients showed no response to conservative management (ma, they were included in the study.

\section{Inclusion criteria of patient selection}

- Pain located in the affected TMJ, especially during opening.

- Joint noises.

- Limited mouth opening (less than $35 \mathrm{~mm}$ ).

- Impeded lateral movement toward the unaffected side.

- Deviation toward the affected side in opening and protrusion movements.

- Patient who did not respond to conservative management including splint therapy.

\section{Exclusion criteria included}

- Patients suffering from any systemic diseases, platelets function disorders, fibrinogen deficiency (18).

- Patients with previous TMJ surgery.

- Patients with previous joint fractures, infection.

- Patients receiving anticoagulation treatment or nonsteroidal anti-inflammatory drugs within 48 hours preoperatively (19), corticosteroid injection at treatment site within 1 month or systemic use of corticosteroids within 2 weeks (18).

\section{Patients were divided into 2 groups}

- Group I: Contained 10 patients who underwent Injection of $2 \mathrm{ml}$ PRP (study group).

- Group II: Contained 10 patients who underwent conventional arthrocentesis (control group).

\section{I.Preoperative phase}

1) Clinical diagnosis

1. Patient questionnaire: all details were recorded in a questionnaire by the examiner including: Chief complaint, Personal data, past history.

2. Clinical examination: general body examination, evaluation of mandibular range of motion, presence of joint sounds, dental evaluation, masticatory muscles tenderness.

3. Panoramic x-ray was done to exclude dental cause of pain.

2) Occlusal splint therapy: hard, clear, silicon, full vacuum splints $2 \mathrm{ml}$ in thickness were constructed for all patients and each patient was instructed to wear the occlusal splint during day time and at night during sleep for four weeks.

3) Complete blood picture was done to ensure the counts of platelets were within normal limits.

\section{II.Operative phase}

In both groups, the same technique of anesthesia was done after disinfection by Povidone-iodine (Betadine ${ }^{\circledR}$, Nile Company, Cairo, Egypt). Canthotragal line was drawn (fig.1). Few drops of local anesthesia (Mepivican with adrenaline, Alexandria Co., Alexandria, Egypt) were injected subcutaneously to block auriculotemporal nerve.

\section{Study group:}

- PRP was prepared by collecting $10 \mathrm{ml}$ blood from the ulnar vein of the patient in a glass centrifuge tube with sodium citrate $(3.2 \%)$ as an anticoagulant under sterile aseptic condition (20).

- The collected blood was mixed with the citrate using rotational movements; an even number of tubes was placed in a centrifuge machine.
- Centrifugation parameters were set to 3,200 rpm, and the centrifugation time was 12 minutes. After separation of the erythrocytes and the platelet poor plasma.

- PRP was layered directly above the erythrocytes, and was aspirated with caution into a separate syringe.

- Activation of PRP by adding $0.4 \mathrm{ml}$ calcium chloride (concentration 10\%) to $2 \mathrm{ml}$ PRP before injection (15).

- The PRP was injected into the upper joint space; the point of injection was located along the canthotragal line, $10 \mathrm{~mm}$ from the middle of the tragus and $2 \mathrm{~mm}$ below the line (fig.2).

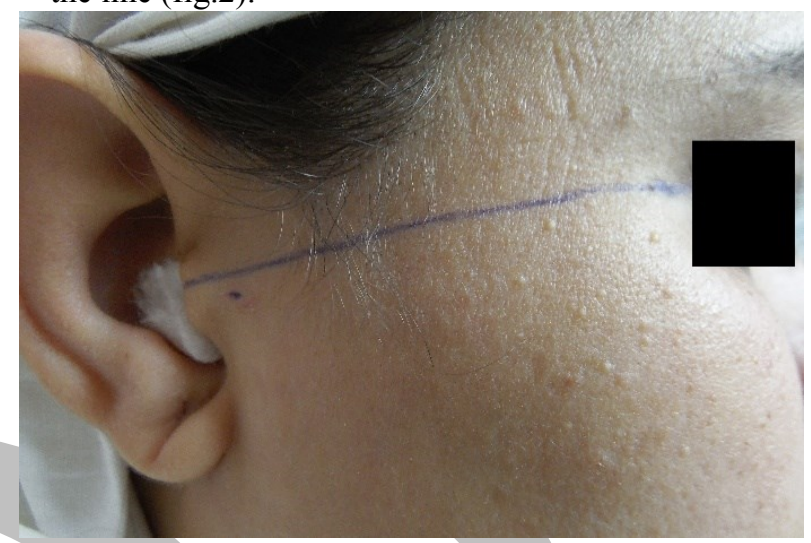

Figure (1): Canthal-tragus line and point of injection PRP

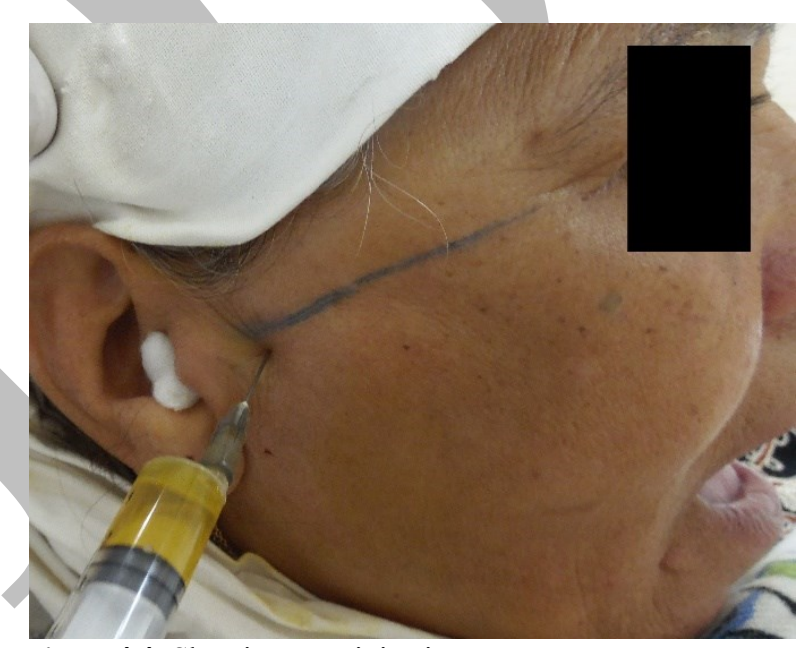

Figure (2): Showing PRP injection

\section{Control group:}

- 20- Gauge needle was inserted into the superior joint space at the glenoid fossa

- Approximately $2 \mathrm{ml}$ of Ringer's lactate solution (Ringer's lactate, Egypt Otsuka Company, Cairo, Egypt) was then injected to distend the superior joint space.

- A second 20-guage needle was inserted into the distended compartment in the area of articular eminence to establish a free flow of the solution through the superior joint space. This needle provides an outflow for the solution which was collected in a kidney dish (fig.3).

- A total of $100 \mathrm{ml}$ of solution was used to lavage the superior joint space, during which time the outlet needle was momentarily blocked with finger pressure 2or 3 times to help distend and break up the joint adhesions.

- Once the needles were removed, the patient's jaw was gently manipulated in the vertical, protrusive and lateral excursions. 


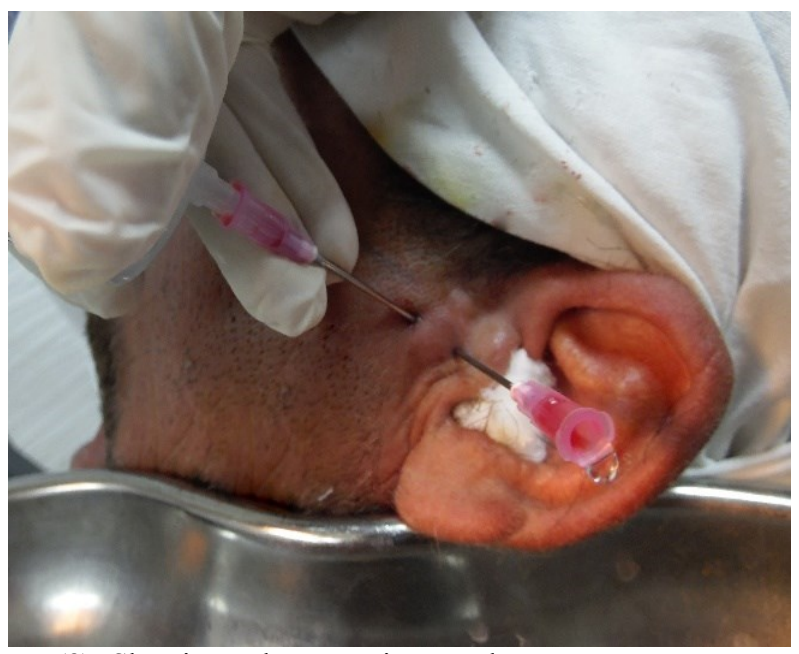

Figure (3): Showing arthrocentesis procedure

\section{The Post-Operative Phase}

- Oral soft diet for 3 days and gradually resume to normal diet after 3 days.

- Post-operative analgesic Paracetamol (Paracetamol 500mg tablet, Sedico Company, Giza, Egypt) one tablet every 8 hrs for 3 days.

- Amoxicillin/clavulanate potassium 1g tablet (Augmentin 1g, GlaxoSmithKline. Cairo, Egypt) one tablet every 12 hrs for 5 days to protect against any possibility of infection.

- Ice packs application.

\section{Follow-up Phase}

Follow up was done at 1 week, 3 months and 6 month intervals.

Postoperative evaluation includes the following parameters:

- Pain level using Visual analogue scale (VAS 1) from zero to ten.

- Maximum interincisal opening measured by the distance in millimeters between the incisal edges of the upper and lower central incisors in vertical direction at maximum pain free mouth opening by using caliper.

- Range of lateral and protrusive movements measured by the distance in millimeters between the upper and lower midlines in horizontal direction on lateral and protrusive movements by using caliper.

- Presence of joint noise at opening and closing was evaluated by light bilateral digital palpation during jaw movement or by stereoscope.

- Presence or absence of joint tenderness on palpation: recorded as tender or not tender.

\section{STATISTICAL ANALYSIS OF THE DATA}

The data were analysed using IBM SPSS software package version 20.0. quantative data was described using range Quantitative data were described using range (minimum and maximum), mean, standard deviation and median and post hoc ANOVA Friedmana statistical significance test.

\section{RESULTS}

The present study has been conducted on twenty patients selected from those attending the outpatient clinic of the Oral and maxillofacial Surgery Department, Faculty of Dentistry, Alexandria University.

Patients were divided into two groups each consisting of ten patients. Group (I) included ten patients; all patients were females, their age ranged from 25 to 50 years (with a mean of $33.8 \pm 8.05$ ). Group (II) included ten patients; eight females and two males, their age ranged from 26 to 42 years (with a mean of $31.7 \pm 5.38$ ).

All patients were evaluated by clinical diagnosis that was made on the basis of RDC/TMD questionnaire axis I survey. All patients in both groups were followed up for 6 months. The duration of the presenting symptoms ranged from 3 months to 10 years.

No major adverse events related to PRP injections or arthrocentesis were observed during treatment and followup period. For group I Patients presented with mild pain and swelling at the site of the injection for 2 days after injection which resolved by itself without any intervention \& for group II mild swelling and tenderness lasted for one week and also it resolved by itself. There were no sign of infection or restriction of the joint movement for both groups.

The statistical analysis of pain intensity (fig.4) (table.1) data showed significant decrease between the preoperative pain score comparing with 1 week, 3 months and 6 months in group I and group II while comparing the two groups there was no statistically significant difference in the pain intensity between both groups during intervals period $(\mathrm{p}>$ $0.05)$.

Regarding the maximum mouth opening, there was a preoperative limited mouth opening (table.2) but the mouth opening (MO) improved postoperatively. The maximum mouth opening scores at 1 week, 3 months, and 6 months are shown in table (2) (fig.5). The mouth opening increased in both groups throughout the follow-up periods with no statistical significant difference $(\mathrm{p}>0.05)$.

The lateral movement (towards the unaffected side) was measured in groups I \& II. They were increased in group I \& group II throughout the follow up periods, but the difference between the two groups throughout the whole follow-up period was found to be statistically insignificant $(p>0.05)$.

Regarding protrusive movement, protrusive movement increased from preoperative to 1 week, 3 months and 6 months postoperative in group I and also in group II but the difference between the two groups throughout the whole follow-up period was found to be statistically insignificant $(\mathrm{p}>0.05)$.

Regarding to TMJ clicking in group I Seven of the ten patients $(70 \%)$ had detectable joint sounds, this decreased to five patients out of ten $(50 \%)$ by the end of the 6 months' follow-up period. There was no significant difference when comparing joint sounds all over the follow up period in this group $(\mathrm{p}>0.05)$.

In group (II) eight of the ten patients (80\%) had detectable joint sounds. By the end of the 6 months' follow-up period, two patients were still complaining of clicking. There was a significant difference when comparing preoperative values with 1 week $(\mathrm{p}=0.031)$ follow up period.

Comparing the two groups There was no statistically significant difference between the two groups regarding TMJ clicking preoperatively and during the postoperative follow- up period $(\mathrm{p}>0.05)$

All patients of the group I \& II had joint tenderness preoperatively. Although there was statistically significant difference between the two groups after 1 week $(p<0.05)$, there was no statistically significant difference between the two groups preoperatively and during the follow- up period at 3 and 6 months $(\mathrm{p}>0.05)$ 
Table (1): Comparison between the two study groups according to pain intensity

\begin{tabular}{|c|c|c|c|c|}
\hline & preoperative & 1 week & 3 months & 6months \\
\hline Group I & & & & \\
\hline $\begin{array}{c}\text { Min. - } \\
\text { Max. }\end{array}$ & $4.0-10.0$ & $1.0-4.0$ & $0.0-2.0$ & $0.0-1.0$ \\
\hline $\begin{array}{c}\text { Mean } \pm \\
\text { SD }\end{array}$ & $6.90 \pm 1.57$ & $2.60 \pm 1.11$ & $0.60 \pm 0.66$ & $0.50 \pm 0.50$ \\
\hline Median & 7.0 & 2.5 & 0.5 & 0.5 \\
\hline $\mathbf{p}_{1}$ & & $0.001^{*}$ & $0.001^{*}$ & $0.001^{*}$ \\
\hline Group II & & & & \\
\hline $\begin{array}{c}\text { Min. - } \\
\text { Max. }\end{array}$ & $5.0-10.0$ & $0.0-4.0$ & $0.0-4.0$ & $0.0-4.0$ \\
\hline $\begin{array}{c}\text { Mean } \pm \\
\text { SD }\end{array}$ & $7.10 \pm 1.97$ & $2.50 \pm 1.20$ & $1.10 \pm 1.20$ & $1.20 \pm 1.46$ \\
\hline Median & 7.50 & 2.5 & 1.0 & 1.0 \\
\hline $\mathbf{P}_{2}$ & & $0.002^{*}$ & $0.002^{*}$ & $0.002^{*}$ \\
\hline $\mathbf{P}$ & 0.813 & 0.849 & 0.263 & 0.169 \\
\hline
\end{tabular}

p1: $\mathrm{p}$ value for Wilcoxon signed ranks test between preoperative and each other periods

$\mathrm{p} 2$ : $\mathrm{p}$ value for Mann Whitney test for comparing between the two studied groups

*: Statistically significant at $\mathrm{p} \leq 0.05$

Table (2): Comparison between the two studied groups according to $\mathrm{MIO}$

\begin{tabular}{|c|c|c|c|c|}
\hline & preoperative & 1 week & $\begin{array}{c}3 \\
\text { months }\end{array}$ & 6 months \\
\hline Group I & & & & \\
\hline $\begin{array}{l}\text { Min. - } \\
\text { Max. }\end{array}$ & & $32.0-50.0$ & $\begin{array}{c}32.0- \\
50.0\end{array}$ & $34.0-50.0$ \\
\hline $\begin{array}{l}\text { Mean } \\
\pm \mathrm{SD}\end{array}$ & $34.30 \pm 8.83$ & $40.50 \pm 8.50$ & $\begin{array}{c}42.50 \pm \\
8.73\end{array}$ & $42.80 \pm 8.28$ \\
\hline Median & 31.0 & 43.0 & 46.0 & 50.0 \\
\hline $\mathbf{p}_{1}$ & & $<0.001^{*}$ & $0.001^{*}$ & $0.001^{*}$ \\
\hline Group II & & & & \\
\hline $\begin{array}{l}\text { Min. - } \\
\text { Max. }\end{array}$ & $10.0-50.0$ & $35.0-51.0$ & $\begin{array}{c}37.0- \\
51.0\end{array}$ & $37.0-53.0$ \\
\hline $\begin{array}{l}\text { Mean } \\
\pm \mathrm{SD}\end{array}$ & $\begin{array}{c}34.20 \pm \\
12.62\end{array}$ & $43.20 \pm 5.87$ & $\begin{array}{c}44.60 \pm \\
5.87\end{array}$ & $45.50 \pm 6.24$ \\
\hline Median & 33.50 & 42.50 & 45.0 & 45.0 \\
\hline $\mathbf{p}_{1}$ & & $0.006^{*}$ & $0.004^{*}$ & $0.003^{*}$ \\
\hline $\mathbf{P}_{2}$ & 0.855 & 0.928 & 0.790 & 0.696 \\
\hline
\end{tabular}

$\mathrm{p}_{1}$ : $\mathrm{p}$ value for Wilcoxon signed ranks test between preoperative and each other periods

$\mathrm{p}_{2}$ : $\mathrm{p}$ value for Mann Whitney test for comparing between the two studied groups

*: Statistically significant at $\mathrm{p} \leq 0.05$



Figure (4): Comparison between the scores of the two studied groups according to pain intensity

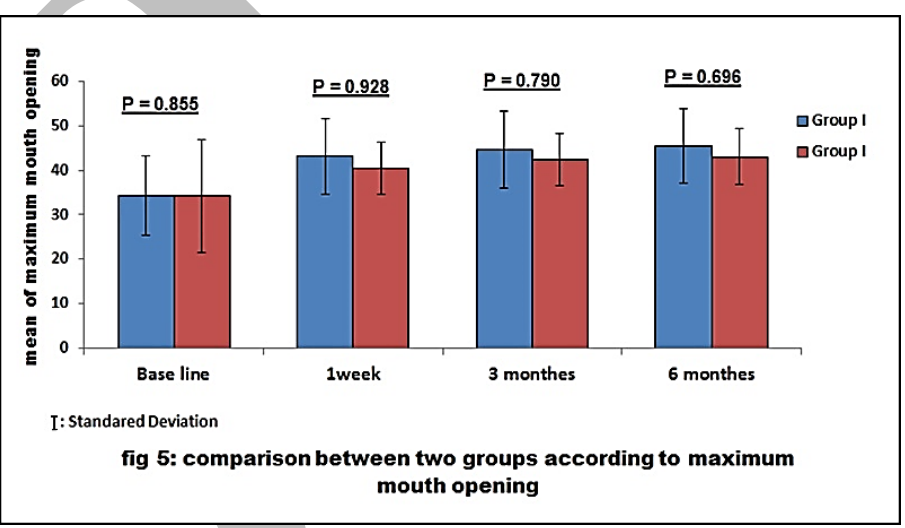

Figure (5): Comparison between the scores of the two studied groups according to MIO

\section{DISCUSSION}

TMDs are important oral health problems which reduce the quality of life of its sufferers. TMD comprise a number of clinical conditions that involve the masticatory musculature and/or TMJ and associated structures (21). TMD pain is the most frequently occurring, non-dental, chronic pain condition in the orofacial area (22).

In this study eighteen patients were females \& two patients were males with age range varied from 25 to 50 years with a mean age range of $33.8 \pm 8.05$. In agreement with many studies which concluded that women had a significantly higher prevalence of clinical signs (23-25). In other studies, female sex seemed to be an increased risk factor for TMD pain (26).

However, there is a difference between these two methods. The purpose of arthrocentesis is to reduce the negative pressure in disc through the lavage and wash out the inflammatory mediators $(24,25)$. On the other hand, PRP injection focuses on the induction of functional recovery by means of regenerating weakened tissues (27), and its anabolic effect on synoviocytes lead to restore HA levels there by enhancing cartilage protection and joint lubrication (11-15).

This reduction in pain in group (I) is in agreement with the results obtained by several authors $(9,14-16,28-34)$ who reported an improvement in the pain levels in their studies after injection of PRP.

Pain decrease after injection of PRP was shown to be related to: early release of protease activated receptor 4 peptides from alpha granule in the platelets which has 
analgesic and anti-inflammatory effect lead to early relief of pain $(14,15)$.

The reduction in pain in group (II) is in agreement with the results obtained by several authors $(24,25,35,36)$ who reported an improvement in the pain level post arthrocentesis in their studies by washing out of inflammatory mediators by arthrocentesis which had its effect in pain reduction and increasing range of movement.

The improvement in mandibular movement in group (I) is in agreement with the results obtained by several authors $(10,15,16,20,33,34,36)$ who reported an increase in the measurements of mandibular movements including maximal interincisal opening, lateral and protrusive movements in their studies after injection of PRP.

Nitzan et al 1991 (25) reported that arthrocentesis proved to be highly effective in providing significant improvement in pain reduction, maximal mouth opening and lateral movement toward the unaffected side in all patients. They claimed that physiotherapy following such treatment produced further improvement which comes in accordance with the results of group (II). In addition, many authors reported the improvement of mandibular functions following arthrocentesis (24-27,34,35).

All patients in both groups had regional (joint and muscle) tenderness preoperatively which decreased gradually along the study period. The results were obtained in group I as the same results were obtained in the other studies $(15,16,32)$. The results in group II are in agreement with the results obtained by several authors (23-25).

However, many studies showed improvement of clicking after one injection of PRP (14-16) patients in this study showed less improvement as in the study of Moon et al. 2014 (35) who found PRP was effective in the treatment of TMJ pain and restricted mouth opening but it was not effective in the treatment of clicking.

These finding of group (II) goes in line with the results obtained by Nitzan et al 1991 (26) who reported that thirteen of seventeen patients did not have clicking after arthrocentesis. It was also in agreement with Yoda et al (37) who found that nine of the twelve joints showed improvement in clicking after arthrocentesis

In the present study, when comparing the clinical outcomes to the preoperative values, both groups showed improvement in all the measured parameters except clicking in group (I) showed less improvement. When comparing between the two groups outcomes, it was found to be insignificant thus, demonstrating the effectiveness of both methods in treatment of TMD patients.

\section{CONCLUSIONS}

From the results of this study we can conclude that: Conservative therapy is very important as initial treatment of TMD, arthrocentesis is recommended as a simple alternative procedure to more invasive TMJ procedures, and PRP intra articular injection is safe and useful for treatment of TMDs.

Further studies are required to confirm the adequate dosage of PRP and the frequency of the injections required to achieve long term results and combination between PRP injection and other modalities.

\section{CONFLICT OF INTEREST}

No potential conflict of interest relevant to this article was reported.

\section{REFERENCES}

1. Miloro M, Ghali GE, Peter EL, Peter DW. Peterson's Principles of Oral and Maxillofacial Surgery. 2nd ed. London: BC Decker Inc Hamilton; 2004.

2. Cooper BC, Kleinberg I. Establishment of a temporomandibular physiological state with neuromuscular orthosis treatment affects reduction of TMD symptoms in 313 patients. Cranio 2008; 26: 104-17.

3. Manfredini, D, Marini M, Pavan C, Pavan L, GuardaNardini L. Psychosocial profiles of painful TMD patients. J Oral Rehabil 2009; 36:193-8.

4. Andersson L, Kahnberg K, Pogrel MA. Oral and Maxillofacial Surgery.UK: Wiley-Blackwell; 2011.

5. Burnouf T, Goubran HA, Chen TM, El-Ekiaby M, Radosevic. Blood-derived biomaterials and platelet growth factors in regenerative medicine. Blood Reviews 2013; 27:77-89.

6. Ferrari M, Zia S, Valbonesi M, Henriquet F, Venere G, Spagnolo $\mathrm{S}$, et al. A new technique for hemodilution, preparation of autologous platelet-rich plasma and intraoperative blood salvage in cardiac surgery.Int J Artif Organs. 1987; 10: 47-50.

7. Brennan PA, Ilankovan V. Arthrocentesis for temporomandibular joint pain dysfunction syndrome. J Oral Maxillofac Surg 2006; 64: 949-51.

8. Lana J, Weglein A, Vicente E, Perez A, Rodrigues A, Luzo $\hat{A}$ et al. Platelet-rich Plasma: Regenerative Medicine: Sports Medicine, Orthopedic, and Recovery of Musculoskeletal Injuries. New York: Springer Science\& Business Media; 2014.

9. Anitua E. Plasma rich in growth factors: Preliminary result of use in preparation of future sites of implants. Int J Oral Maxillofac Implants 1999; 14:529-35.

10. Cerza F, Carnì S, Carcangiu A, Di Vavo I, Schiavilla V, Pecora, A, et al. Comparison between hyaluronic acid and platelet-rich plasma, intra-articular infiltration in the treatment of gonarthrosis. Am. J. Sports Med 2012; 40: 2822-7.

11. Akeda K, An H, Okuma M, Attawia M, Miyamoto K, Thonar E, et al. Platelet-rich plasma stimulates porcine articular chondrocyte proliferation. Osteoarthritis and Cartilage Journal 2006; 14:1272-80.

12. Xie X, Zhang C, Tuan RS. Biology of platelet-rich plasma and its clinical application in cartilage repair and matrix biosynthesis. Arthritis Research \& Therapy 2014; 16:20419.

13. Mazzocca AD, Mc Carthy BR, Intravia J, Beitzel K, Apostolakos J, Cote MP, et al. An in vitro evaluation of the anti-inflammatory effects of platelet-rich plasma, ketorolac, and methyl prednisolone. J Arthroscopy 2013; 29:675-83.

14. Van Osch GJ, Bernsen MR, van Buul GM, Koevoet WL, Kops N, Bos PK, Verhaar JA, et al. Platelet-rich plasma releasate inhibits inflammatory processes in osteoarthritic chondrocytes. Am J Sports Med 2011; 39:2362-70.

15. Machoň V, Řehořová M, Šedý J, Foltán R. Platelet-Rich Plasma in Temporomandibular Joint Osteoarthritis Therapy: A 3-Month Follow-Up Pilot Study. J Arthritis 2013; 2: 112.

16. Pihut M, Szuta M, Ferendiuk E, Zeńczak, Więckiewicz D. Evaluation of Pain Regression in Patients with Temporomandibular Dysfunction Treated by IntraArticular Platelet-Rich Plasma Injections: A Preliminary Report. Biomed Res Int 2014; 2014:132369. 
17. Hall, Michael P, Band P, Meislin, R, Jazrawi L, Cardone D. Platelet-rich plasma: Current concepts and application in sports medicine. JAAOS 2009; 17: 602-8.

18. Cantu R, Steffe J. Rehabilitation: for the post-surgical orthopedic patient. In Soft tissue healing considerations after surgery.3rd ed. St Louis: Mosby; 2001.

19. Lana J. F, Weglein A, Vicente E, Perez A, Rodrigues A, Luzo Â. C. et al. Platelet-rich Plasma: Regenerative Medicine: Sports Medicine, Orthopedic, and Recovery of Musculoskeletal Injuries. New York: Springer Science\& Business; 2013.

20. Sharma S.M, Dhruvit T. clinical efficacy of autologous concentrate platelets in treatment of TMJ disorders- apilot study. Nitte University J of Health Science .2014; 4:70-4.

21. De Leeuw, Reny, and Gary D. Klasser. Orofacial pain: guidelines for assessment, diagnosis, and management. 4th ed. Chicago: Quintessence; 2008.

22. Tozoglu, S, Al-Belasy F A, and Dolwick M. A. review of techniques of lysis and lavage of the TMJ. Br J Oral Maxillofac Surg 2010; 3:1-8.

23. ELNager Y, Shawky N, Ragaey H, Abd El-latief G. the study of the effect of arthocentesis on muscle activiy using electromyogram. Theses for master degree in Oral \& Maxillofacial Surgery. Alexandria University, 2010.

24. EL-said S, Shawky N, Ragaey H. Comparative study of arthrocentesis with or without using piroxicam in the management of temporomandibular joint disorders. Theses for master degree in Oral \& Maxillofacial Surgery. Alexandria University, 2015.

25. Nitzan DW, Dolwick MF, Martinez GA. Temporomandibular joint arthrocentesis: a simplified treatment for severe, limited mouth opening. J Oral Maxillofac Surg 1991; 49: 1163-7.

26. Botelho A, De Arruda Veiga M. Influence of sex on temporomandibular disorder pain: a review of occurrence and development. Braz J Oral Sci 2008; 7: 1631-5.

27. Yoda T, Imai H, Shinjyo Y, Sakamoto I, Abe M, Enomoto $\mathrm{S}$. Effect of arthrocentesis on TMJ disturbance of mouth closure with loud clicking: a preliminary study. J Craniomandibular Pract 2002; 20: 18-22.

28. Anitua E. Plasma rich in growth factors: Preliminary result of use in preparation of future sites of implants. Int J Oral Maxillofac Implants 1999; 14:529-35.

29. Chomicki P, Zakrzewski P, Pomianowski S, Bindas P. Platelet concentrates, as new and promising agent in the orthopedic surgery- an introduction. Progres of Medical Science 2010; 23: 153-7.

30. Ficek K, Ficek A. Platelet rich plasma application in a bone loss case. Medical Cases 2013; 4: 175-9.

31. Iwanaga T, Shikichi M, Kitamura H, Yanase H, NozawaInoue K. Morphology and functional roles of synoviocytes in the joint. Arch Histol Cytol. 2000; 63:17-31.

32. Hegab AF, Ali HE, Elmasry M, Khallaf MG. Platelet-Rich Plasma Injection as an Effective Treatment for Temporomandibular Joint Osteoarthritis. J Oral Maxillofac Surg 2015; 73:1706-13.

33. Hanci M, Karamese M, Tosun Z, Aktan T, Duman S, Savaci $\mathrm{N}$. Intra-articular platelet-rich plasma injection for the treatment of temporomandibular disorders and a comparison with arthrocentesis. J Craniomaxillofac Surg 2015; 43:162-6.

34. Moon S, Lee S, Ryu J. Ultrasound-guided Platelet-rich Plasma Prolotherapy for Temporomandibular Disorders. J Oral Med Pain 2014; 39: 140-5.
35. Alpaslan GH, Alpaslan C. Efficacy of temporomandibular joint arthrocentesis with and without injection of sodium hyaluronate in the treatment of internal derangements. J Oral Maxillofac Surg 2001; 59:613-8.

36. Simsek M. Bilateral platelet rich plasma injections with assisted techniques for temporomandibular joint disorders. Eur Res J 2016; 2: 42-5. 